\title{
Traduire
}

Revue française de la traduction

$230 \mid 2014$

À la croisée du texte et de l'image

\section{Entretien avec Jean-François Cornu}

Samuel Bréan, Anne-Lise Weidmann et Jean-François Cornu

\section{(2) OpenEdition}

Journals

Édition électronique

URL : http://journals.openedition.org/traduire/614

DOI : 10.4000/traduire.614

ISSN : 2272-9992

Éditeur

Société française des traducteurs

Édition imprimée

Date de publication : 15 juin 2014

Pagination : 10-21

ISSN : 0395-773X

\section{Référence électronique}

Samuel Bréan, Anne-Lise Weidmann et Jean-François Cornu, «Entretien avec Jean-François Cornu », Traduire [En ligne], 230 | 2014, mis en ligne le 15 juin 2016, consulté le 01 mai 2019. URL : http:// journals.openedition.org/traduire/614; DOI : 10.4000/traduire.614 


\title{
Entretien avec Jean-François Cornu
}

\author{
Propos recueillis par Samuel Bréan et Anne-Lise Weidmann \\ le 25 janvier 2014
}

Jean-François Cornu traduit de l'anglais pour l'audiovisuel et l'édition depuis 1985 et mène parallèlement des recherches sur la traduction/adaptation audiovisuelle. Son ouvrage consacré à l'histoire et à l'esthétique du doublage et du sous-titrage(1) retrace l'évolution des deux grands modes de traduction du 7e art, depuis l'apparition du cinéma parlant à la fin des années 1920. Il évoque dans cet entretien sa démarche et le fruit de ses recherches.

Le doublage et le sous-titrage. Histoire et esthétique paraîtra prochainement aux Presses universitaires de Rennes. À qui s'adresse cet ouvrage?

Dès les phases initiales de ma recherche pour la thèse de doctorat, dont il est une version enrichie et mise à jour, j'ai eu l'intention d'en faire un texte accessible à n'importe quel lecteur s'intéressant au cinéma et susceptible de lire, de la même manière, un ouvrage sur le montage, le métier de chef opérateur, la réalisation, etc. J'ai cherché à raconter une histoire, tant dans la partie historique qui s'y prête particulièrement bien, que dans celle, plus personnelle, qui aborde la dimension esthétique du doublage et du sous-titrage. La collection des Presses universitaires de Rennes dans laquelle il va paraître, "Le Spectaculaire/Cinéma ", a déjà à son actif quelques titres spécialement consacrés au son et à la parole au cinéma.

Quel a été le point de départ de vos recherches?

J'ai commencé à pratiquer le sous-titrage au milieu des années 1980 et, dès le départ, j'ai eu envie de savoir comment tout cela avait commencé. Dans les histoires du cinéma qui existaient à l'époque, on apprenait qu'il était devenu parlant presque du jour au lendemain, et qu'à partir du moment où les langues étaient devenues un obstacle pour la diffusion internationale des films, on avait inventé le doublage et le sous-titrage. Ça s'arrêtait là ! En creusant un peu, j'ai constaté que ces pratiques étaient le fruit d'une évolution étalée sur plusieurs années ; même si, rétrospectivement, tout s'est fait assez rapidement, les choses ne sont pas tombées du ciel.

(1) CORNU, Jean-François, Le doublage et le sous-titrage. Histoire et esthétique, Presses universitaires de Rennes, à paraître. 
Les études plus universitaires sur la question étaient rares et mettaient de façon exclusive l'accent sur le texte, le contenu des sous-titrages et des doublages et les analyses comparatives, comme on le fait pour étudier la traduction littéraire. Je me suis rendu compte que c'était finalement assez vain et que cela ne permettait guère d'aller au-delà d'un jugement du type : "C'est bien traduit ou c'est mal traduit, pour telle raison ». J'étais beaucoup plus intéressé par le point de vue de l'histoire du cinéma sur la transition du muet vers le parlant, qui me paraissait être un point aveugle, un vide dans l'histoire du cinéma. J'ai voulu adopter une démarche plus cinéphilique sur l'histoire du doublage et du sous-titrage.

En effet, le texte de la traduction n'est pas un texte isolé que l'on ne pourrait étudier que comparativement au texte original des dialogues. J'ai souhaité mettre l'accent sur une dimension qui paraît aussi incontournable que naturelle quand on pratique soi-même la traduction audiovisuelle, à savoir la prise en compte simultanée du texte original, de l'image et du son, qui sont liés de façon inextricable. C'est là, à mon avis, l'erreur et l'impasse des traductologues qui n'ont pas su prendre en compte cette interdépendance. Puisqu'on en tient compte sans même y penser lorsque l'on pratique ce type de traduction, il m'a paru indispensable d'adopter la même approche pour m'y intéresser du point de vue de l'histoire et de l'esthétique.

Le doublage et le sous-titrage ne sont donc pas apparus du jour au lendemain. Comment est né le doublage, pour commencer?

Rappelons qu'en Europe, 1929 est une année cruciale. Le Chanteur de jazz (The Jazz Singer, Alan Crosland, 1927) arrive sur les écrans américains fin 1927, mais c'est à partir de 1929 que les choses évoluent de façon significative du point de vue du doublage. Elles se stabilisent vraiment à partir de 1933-1934 dans la plupart des pays d'Europe.

Les prémices du doublage apparaissent à la fois aux États-Unis et en Allemagne, ainsi qu'en France, dans une moindre mesure. Ce sont initialement des nécessités commerciales qui président à la naissance du doublage : il faut trouver le moyen de surmonter la barrière des langues qui apparaît avec le cinéma parlant. Curieusement, les studios hollywoodiens n'avaient pas anticipé ce problème. Soudain, c'était la panique à bord! À côté des rudiments de soustitrage et de doublage, ils ont exploré un temps la piste des "versions multiples ", c'est-à-dire le tournage de plusieurs versions d'un même film, en différentes langues et avec des acteurs venant de différents pays. En Allemagne, qui était la grande cinématographie en Europe à l'époque, du moins du point de vue du nombre de films produits, la situation était à peu près identique, même si la réaction a été moins affolée.

À partir de la fin de l'année 1930 et surtout de 1931, les Américains ont eu l'idée d'adjoindre d'autres voix aux corps des acteurs que l'on voyait à l'écran. Cela s'est fait sous une forme imaginée, sous l'influence probable de techniciens allemands, par Claude Autant-Lara qui n'était pas encore cinéaste, mais réalisateur de versions multiples chez MGM : faire mimer par les acteurs originaux ce qu'ils devraient dire dans les autres langues (français, espagnol et 
allemand, notamment) et enregistrer simultanément les voix françaises avec des comédiens hors champ. Cette forme de doublage ne portait pas de nom officiel à l'époque, mais on l'appelle généralement " doublage en direct ». Même s'il ne s'agit pas d'un doublage à proprement parler, on peut en voir une illustration dans la version parlante de Chantage d'Alfred Hitchcock (Blackmail, 1929), où la comédienne principale (Anny Ondra, de langues tchèque et allemande) mime ses paroles en anglais, tandis que la voix qu'on entend est celle d'une comédienne anglaise. Dans ce cas précis, il n'y a pas de changement de langue en jeu, mais le principe est le même.

Très vite, les Américains se sont rendu compte que ce procédé ne permettait pas d'obtenir un synchronisme satisfaisant et qu'il nécessitait en outre de simplifier le dialogue par rapport aux répliques originales. Claude Autant-Lara raconte dans ses souvenirs(2) qu'il a ensuite eu l'idée d'une autre méthode : conserver l'image originale, avec les mouvements de bouches correspondant à la langue anglaise, et y associer des voix après coup, dans une nouvelle phase qu'on a baptisée postsynchronisation en français, mais que les Américains appelaient alors "dubbing".

II restait à synchroniser le texte français avec les mouvements de bouches de la version originale. Dans la méthode américaine, on procédait initialement par " approximations successives " (l'expression est celle d'un critique de La Cinématographie française). À force de répétitions, de visionnements des scènes, de tâtonnements, on cherchait une certaine adéquation des textes dits par les comédiens aux mouvements labiaux, de façon plus ou moins précise, et surtout aux attitudes et expressions des personnages. Les comédiens répétaient à l'avance pour apprendre leur texte par cœur, même s'il s'agissait de courtes scènes. Ils ne disposaient d'aucun " guide " ni repère pour l'enregistrement.

Cette méthode " américaine " a été appliquée en France. En effet, lorsque la législation française a imposé, en 1932, que les doublages soient réalisés sur le territoire national, les pionniers français qui exerçaient à Hollywood sont revenus en France et ont perpétué cet usage que l'on a fini par appeler "doublage à l'image ", puisque les comédiens étaient uniquement guidés par l'image. Cela a perduré jusqu'au milieu des années 1960, tant que les majors américaines ont eu des auditoriums de doublage à Paris.

L'autre méthode des débuts, la méthode " allemande ", reposait sur des éléments techniques et mécaniques, plus rigoureux. Mise au point à Berlin par Carl Robert Blum en 1930, elle s'inspirait d'un système de synchronisation de la musique pour les films muets : il s'agissait de transcrire le rythme visuel d'un film sur une bande où étaient consignés des signes indiquant les changements de plans, le rythme du montage ou, parfois, certains éléments de l'image. Le compositeur chargé de créer une musique originale pouvait ainsi trouver une correspondance

(2) Claude Autant-Lara, Hollywood Cake-Walk (1930-1932), Paris, Henri Veyrier, 1985. 
musicale au rythme des images. Ce procédé a naturellement trouvé une application pour la synchronisation des voix. Il a ensuite été adopté en France sous le nom de "Rythmographie ", qui est devenu à la fois le nom du système et de l'entreprise qui l'exploitait commercialement. II consiste à reporter les mouvements de bouches - ouvertures, fermetures, silences... - sous la forme de signes codés sur une bande qui a pris le nom générique de "bande rythmo " au fil du temps, indépendamment du procédé initial. Aujourd'hui, peu de gens savent quelle est l'origine de la bande rythmo. Un ingénieur français, Charles Delacommune, avait mis au point un système similaire, le "Ciné-Pupitre ", pour la synchronisation tant de la musique que des voix.

\section{Qui sont les premiers auteurs de doublage?}

II est très difficile de le savoir. Les traces que l'on trouve concernent plutôt les comédiens, les conditions d'enregistrement et la création de différents procédés, mais on peine à cerner la place des traducteurs, en tout cas pour les deux ou trois premières années.

De plus, il est difficile d'avoir accès aujourd'hui à ces premières versions doublées. Le film que je cite toujours, par la force des choses, est Grand Hôtel (Grand Hotel) d'Edmund Goulding, réalisé en 1932, qui a été édité en DVD avec sa version doublée d'origine. II est particulièrement intéressant, car c'est l'un des tout derniers doublages français réalisés aux États-Unis, avant l'adoption de la législation imposant qu'ils soient faits sur le territoire français. II nous donne un témoignage visuel et auditif du travail des comédiens. La méthode est celle de Claude Autant-Lara, même si c'est Henri de la Falaise qui a dirigé ce doublage. Toutefois, je n'ai pas encore réussi à trouver qui avait travaillé sur l'adaptation des dialogues, ni si on était parti d'une traduction intégrale et littérale qui avait ensuite été adaptée pour être jouée, etc. Dans les premiers temps du doublage, on entend souvent parler d'une traduction, dont on ne sait jamais qui l'a faite, et ensuite d'une adaptation pour le doublage et parfois pour le sous-titrage.

On sait qu'à partir du moment où le doublage - et le sous-titrage dans une moindre mesure s'est vraiment installé et développé, les distributeurs ont mis en avant l'intervention de " littérateurs ", c'est-à-dire d'écrivains ou de journalistes de renom, qui donnaient au doublage qu'ils signaient une sorte de caution de qualité. Suzanne Chantal et Marcel Duhamel racontent que certains écrivains (comme André Maurois) signaient l'adaptation alors qu'ils n'étaient en réalité pas intervenus dans la traduction ou étaient partis d'une traduction existante qu'ils avaient adaptée. J'ignore si ce sera possible un jour, mais il faudrait essayer de retrouver les anonymes et les sans-grade des tout débuts de la traduction audiovisuelle. Il y a forcément des gens qui sont intervenus à ce moment-là, sans le travail desquels le reste n'aurait pas pu suivre, mais dont on n'a pas trace.

\section{À partir de quel moment y a-t-il eu des " auteurs de doublage " professionnels ?}

Je pense que c'est lié au moment où la législation a rendu obligatoire la traduction des dialogues en France. II a soudain fallu trouver sur place des personnes compétentes qui devaient être à 
la fois bons traducteurs et bons dialoguistes. Les adaptateurs de doublage se recrutaient chez les journalistes et les écrivains, mais aussi chez les jeunes scénaristes et dialoguistes de langue française. Henri Jeanson et Charles Spaak ont ainsi signé quelques doublages à leurs débuts, avant d'écrire des dialogues de films français. On faisait également appel à des journalistes travaillant pour des revues de cinéma : Lucie Derain et Raymond Berner (chroniqueurs de La Cinématographie française, notamment) ont travaillé pour le sous-titrage et, dans une moindre mesure, pour le doublage. Avec, parfois, des positions un peu schizophrènes, puisqu'ils critiquaient le doublage dans leurs écrits, mais en vivaient occasionnellement! On peut considérer que c'est à partir du milieu des années 1930 que des professionnels se sont spécialisés dans ce domaine.

\section{Qu'en est-il des origines du sous-titrage?}

Il y a une filiation entre le sous-titrage et les intertitres du cinéma muet, même si elle est à mon sens plus ténue que l'affirment certains historiens du cinéma. Rappelons que les intertitres ou " cartons " étaient des panneaux de texte fournissant des indications narratives sur le déroulement d'une histoire dans un film de fiction muet et reproduisant une partie des dialogues que les acteurs étaient censés avoir prononcés. Ces intertitres prenaient généralement la forme d'une inscription sur fond noir qui interrompait le déroulement visuel du film.

Vers la fin du muet, certains films ont commencé à mélanger l'image et le texte, sans que ce soit systématique : on voit parfois des textes qui apparaissent en surimpression, sur l'image. C'est par exemple le cas de certains films de Friedrich Wilhelm Murnau. On peut donc considérer qu'il y a des points communs entre ces intertitres tardifs qui se mêlaient à l'image et les premiers sous-titrages. Cependant, la nature des intertitres du muet et des sous-titres du parlant est radicalement différente. La raison d'être des sous-titres tient justement au fait que les films sont désormais parlants ; leur présence est liée à un son audible.

Au fil du temps, on va commencer à tenir compte aussi du rythme de l'image et adopter certains principes, notamment la règle d'or des changements de plan : on s'efforce d'éviter de faire durer un sous-titre sur deux plans successifs, afin que le spectateur ne relise pas le même sous-titre lorsqu'apparaît une nouvelle image. II est d'ailleurs difficile de dater précisément cette règle. Je pense personnellement qu'elle remonte à l'époque où l'on a commencé à faire le repérage (c'est-à-dire le marquage du point d'apparition et du point de disparition de chaque sous-titre) avant la traduction. Dans un film comme Les Tueurs (The Killers, Robert Siodmak, 1946), sous-titré à la fin des années 1940 ou au début des années 1950, on voit bien que le respect des changements de plan n'est pas encore systématique.

Le sous-titrage des débuts est donc fortement guidé par le son...

C'est flagrant dans Jeunes Filles en uniforme (Mädchen in Uniform, Leontine Sagan), un film de 1931 dont les sous-titres français sont signés Colette. Les changements de plan n'ont aucune 


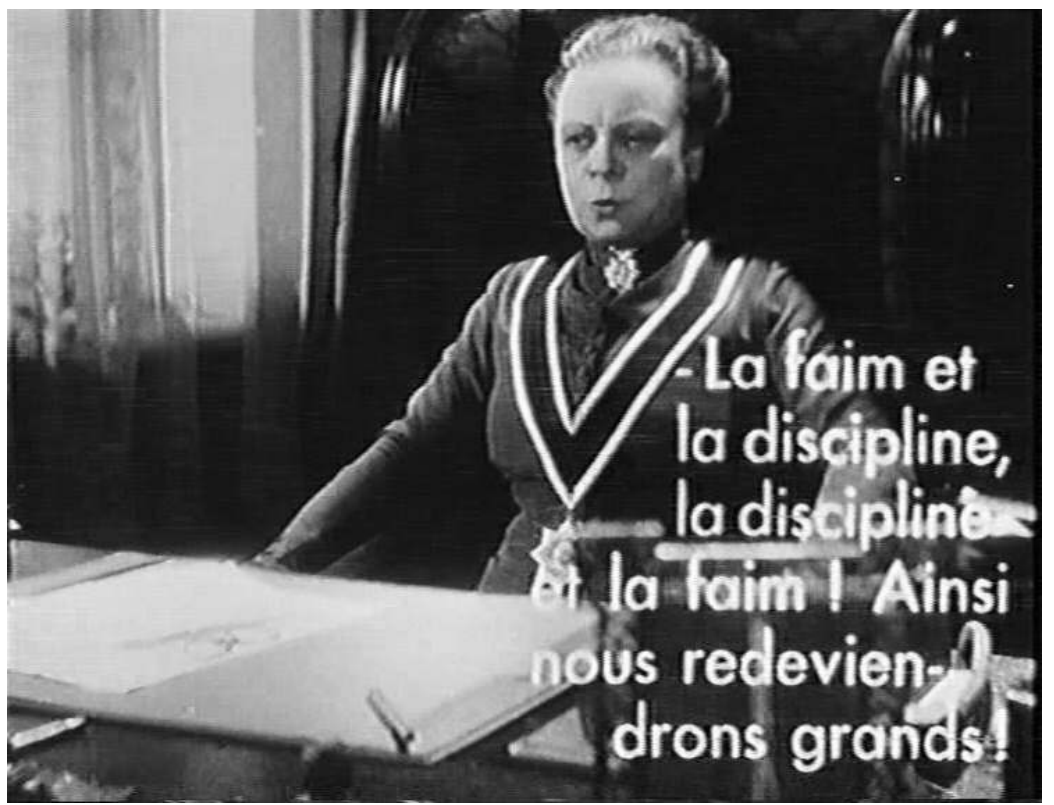

incidence sur le repérage des sous-titres. Ce sous-titrage est intéressant, parce qu'il témoigne d'une volonté de restituer une langue particulière. C'est sans doute là que réside l'apport de Colette, qui n'a pas réalisé elle-même la traduction, mais a adapté une traduction littérale et mis sa patte dans le style et la façon de parler des différents personnages.

Les sous-titres de Jeunes Filles en uniforme sont composés en caractères de grande taille, ce qui tient à l'un des premiers procédés utilisés pour les incruster dans l'image. Mais surtout, ils apparaissent parfois sur plus de deux lignes. En outre, quand des zones claires dans l'image empêchent la lecture de caractères blancs sur fond blanc, la disposition du sous-titre change : il est parfois " en colonne " sur la droite de l'écran, sur des zones plus sombres. II empiète alors beaucoup sur l'image, mais est effectivement plus lisible. On aboutit à des lignes très courtes qui comportent parfois des césures dans les mots : une syllabe suivie d'un trait d'union... toutes choses qui seraient inimaginables aujourd'hui.

De quand date l'adoption de la norme actuelle en la matière, c'est-à-dire des sous-titres de deux lignes maximum au bas de l'écran?

On trouve encore des sous-titres sur trois lignes dans les années 1940, par exemple sur une copie de Scandale à Paris ( $A$ Scandal in Paris, Douglas Sirk, 1946) conservée à la Cinémathèque de Luxembourg. L'adoption des deux lignes dépend des laboratoires de sous-titrage. Chez Titra Film, pionnier du sous-titrage en Europe, la pratique s'est assez vite stabilisée sur deux 
lignes au bas de l'image. II serait intéressant d'avoir accès aux listes de sous-titres de ce laboratoire qui datent des années 1930, si elles ont été conservées, pour voir ce qui se faisait avant la guerre. Quand Cinétitres, le deuxième grand laboratoire de sous-titrage français, a été créé en 1958, il a tout de suite adopté le principe des deux lignes au bas de l'image. On peut donc supposer qu'il s'agissait alors d'une pratique déjà courante.

\section{Comment étaient distribués les films sous-titrés ?}

En 1932, lorsque la législation a rendu obligatoire la réalisation des doublages en France, elle a aussi imposé, au moins de façon théorique, d'assez fortes contraintes sur la diffusion des films en version originale. Officiellement, la diffusion des films en version originale (sous-titrée ou non) était limitée à cinq salles à Paris et cinq salles dans le reste de la France. Cela a été le cas au moins pour toutes les années 1930, jusqu'au début de la guerre. Cette législation réduisait nettement la diffusion des films en VOST. Très peu de films avaient donc besoin d'être sous-titrés et il n'y avait pas beaucoup de travail pour les laboratoires et les adaptateurs.

\section{Quelle était la justification de cette restriction?}

Il est difficile de trouver une explication officielle. II s'agissait probablement de mettre en avant la langue française, mais cet argument n'est indiqué nulle part dans les sources officielles. Sous les régimes fasciste et franquiste, l'Italie et l'Espagne ont adopté des lois pour favoriser le doublage, mais celles-ci avançaient clairement des raisons nationalistes de protection de la langue. En France, aucun texte officiel ne semble mentionner de telles raisons. II y avait cependant de fortes pressions nationalistes dans l'Europe de l'époque et il n'est pas complètement faux d'invoquer ce type de motivations.

Ces dispositions très contraignantes pour la diffusion des VOST ont cependant été rapidement assouplies, moyennant des dérogations. Lorsque l'on consulte la presse de l'époque pour voir quels films étaient à l'affiche, notamment à Paris, on voit bien que les versions originales n'étaient pas restreintes à cinq salles. La contrainte législative n'était donc pas strictement appliquée dans la capitale.

En province, il était plus difficile de passer outre, moins pour des questions de réglementation qu'en raison de la position des exploitants de salles. Ceux-ci affirmaient, à tort ou à raison, que le public des salles de province (même dans les grandes villes) voulait des films qui parlent français et n'avait pas envie de lire des sous-titres.

II est frappant de constater que les choses se sont figées très tôt pour aboutir à la situation que l'on connaît aujourd'hui (la VOST à Paris et dans les grandes villes, la VF partout ailleurs). À ceci près que ce n'était pas la nature des films en eux-mêmes qui déterminait alors le choix d'une exploitation en VOST (films " d'art et d'essai " aujourd'hui pour le sous-titrage) ou en VF, mais plutôt le public visé. Dans la presse cinématographique de l'époque, on parle même du " gros " public, quand aujourd'hui on parle du " grand " public, c'est-à-dire du public " moyen ". 
II faut également préciser que, pendant toutes les années 1930, beaucoup de films étrangers étaient d'abord présentés en version originale à Paris (avec ou sans sous-titres), ce qui servait de test aux distributeurs et permettait de juger de leur réception par le public. Si l'accueil était bon, on n'hésitait pas alors à le doubler pour le plus grand public et le public de province. Aujourd'hui - et c'est le cas depuis longtemps déjà - si un distributeur décide de sortir un film à la fois en version doublée et en version sous-titrée, les deux se font en général simultanément.

II y avait donc un décalage entre la sortie des deux versions. Pour le doublage, les studios américains privilégiaient les films faisant appel à de grandes vedettes. Ils accordaient un soin particulier au doublage, afin que le public le plus large possible puisse apprécier ses vedettes préférées, Marlene Dietrich, Joan Crawford, Ava Gardner... II était important de choisir des voix qui correspondaient bien aux acteurs et de faire écrire de bons dialogues.

Cette habitude de sortir d'abord une VOST, puis une version doublée pour les films à succès, a dû disparaître après la guerre, selon moi. Mais il y aurait encore beaucoup de recherches à faire concernant l'histoire des stratégies commerciales et de la distribution des versions.

À part quelques grands noms comme Colette, en sait-on plus sur les premiers auteurs de sous-titrage?

Les premiers adaptateurs pour le sous-titrage ont le même profil que les premiers dialoguistes de doublage. D'ailleurs, certains faisaient les deux. lls se recrutent dans les mêmes professions : journalistes, scénaristes... Suzanne Chantal, qui était journaliste, a ainsi mené de front une double carrière pendant les années 1930 : elle était à la fois rédactrice en chef de la revue Cinémonde et a sous-titré des films à un rythme assez stupéfiant(3).

\section{Comment ont évolué les techniques liées au sous-titrage?}

Jusqu'à la fin des années 1950, soit pendant près de trois décennies, le sous-titrage se passait comme suit : le traducteur-adaptateur voyait le film une seule fois, lors d'une projection en salle dans le meilleur des cas. II avait à sa disposition une transcription des dialogues originaux, mais devait ensuite se fier à ses seuls souvenirs de la projection. Certains prenaient des notes en projection: André Rigaud (auteur de doublages et de sous-titrages) raconte par exemple que son regard naviguait entre l'image et la transcription des dialogues pendant qu'il effectuait une sorte de découpage des répliques. II se basait ensuite sur ce découpage quelque peu sommaire pour construire ses sous-titres.

Dans un deuxième temps, les sous-titres étaient repérés sur le film, au photogramme près (c'est-à-dire au 1/24e de seconde près), mais le découpage définitif ne pouvait se faire qu'en

(3) Voir par exemple son témoignage dans Suzanne Chantal, Cinémonde, $n^{\circ} 378,16$ janvier 1936, p. 42 et n 379 , 23 janvier 1936, p. 62 ; repris sur le blog de l'ATAA les 5 et 12 février 2012 (www.ataa.fr/blog/tag/suzanne-chantal/). 
fonction de l'image. Cette étape était assurée par des techniciens qui s'efforçaient, à partir du découpage initial fait par les adaptateurs, de faire entrer dans l'image les sous-titres prévus. Le résultat était souvent approximatif : les sous-titres ne commençaient pas exactement au moment où l'on entendait le son sortir de la bouche du personnage et les changements de plan n'étaient pas pris en compte.

Initialement, la traduction précédait le découpage en sous-titres. Ce sont les traducteurs qui ont demandé un changement de méthode aux deux grands laboratoires de sous-titrage à la fin des années 1950. II leur semblait plus facile et plus rigoureux, pour leur travail, que le repérage se fasse en amont, puisqu'il permettait de guider l'adaptation.

Simon Laks était sans doute de ceux-là. Cet auteur de sous-titres a publié en 1957 un essai pionnier sur le sous-titrage dans lequel il insiste sur l'importance d'effectuer un repérage avant la traduction(4). Cela signifie qu'en 1956-1957, au moment probable de la rédaction de son ouvrage, la pratique était encore quelque peu flottante, il devait encore être courant de faire le repérage après la traduction et non avant. On peut ainsi dater assez clairement ce changement.

À partir de cette époque, on s'est donc mis à déterminer à l'avance, à partir des répliques du film, où allaient apparaître les futurs sous-titres. La rédaction des sous-titres par les adaptateurs s'est faite dès lors en fonction de la durée du sous-titre, convertible en un certain nombre de caractères. On a commencé à voir des sous-titrages plus rigoureux du point de vue de l'apparition des sous-titres à l'écran, de leur rythme, de la prise en compte des changements de plans et de la lisibilité. Cela a permis d'affiner les traductions.

La deuxième grande révolution a eu lieu trente ans plus tard, à la fin des années 1980 : il s'agit de l'arrivée de la vidéo et de l'informatique. Je peux en témoigner, car j'ai connu les dernières années du repérage effectué sur table de montage et du visionnage par les adaptateurs sur ces tables, avec un écran et un son de qualité médiocre, bobine par bobine. La vidéo et l'informatique ont apporté un confort sans précédent.

L'évolution de la technique du sous-titrage a donc été plus saccadée que celle du doublage.

Dans le doublage, les choses ont longtemps été plus fluides et, dans l'ensemble, le doublage tel qu'il se pratique aujourd'hui ne diffère guère du doublage des origines. II y a eu des apports techniques, comme l'informatisation, qui ont permis d'affiner certaines étapes, mais qui n'ont pas modifié fondamentalement le processus de réalisation du doublage.

Les bouleversements que l'on connaît sont très récents. Les doublages sont réalisés dans des délais de plus en plus serrés. Mais surtout, des logiciels de doublage sont apparus, des

(4) Simon Laks, Le sous-titrage de films. Sa technique - son esthétique, Paris, Propriété de l'auteur, 1957 ; repris dans L'Écran traduit, hors-série $n^{\circ}$ 1, 2013 (http://ataa.fr/revue/archives/1343). 
" bandes rythmo virtuelles ". II s'agit d'un outil non négligeable, qui conduit cependant à la disparition de certains maillons de la chaîne de doublage. C'est notamment le cas de la phase cruciale de détection en doublage, l'étape technique qui consiste à transcrire sous forme de signes codés les mouvements de bouche des comédiens. Cette tâche est de plus en plus souvent confiée aux adaptateurs, aidés par les logiciels de doublage. De même, la calligraphie et la frappe des textes autrefois écrits à la main disparaissent, puisqu'elles peuvent être réalisées automatiquement.

Le problème ne vient pas des outils en eux-mêmes, mais de l'usage qui en est fait : désormais, on suggère fortement - ou on impose - aux adaptateurs d'accomplir certaines phases de travail qui étaient autrefois assurées par des techniciens, mais sans nécessairement leur accorder une contrepartie pour ces nouvelles tâches.

Le même phénomène se retrouve dans le sous-titrage : la phase du repérage des sous-titres, qui a longtemps été accomplie par des techniciens, est de plus en plus souvent confiée aux adaptateurs, qui disposent de leur propre logiciel. Là encore, cette évolution parfois dommageable pour les adaptateurs ne tient pas aux outils, mais aux pressions des donneurs d'ordre. C'est une très bonne idée de repérer soi-même le film que l'on va adapter. Ce qui est gênant, c'est qu'il s'agit souvent d'une obligation et qu'on ne rémunère pas toujours les adaptateurs pour réaliser ces opérations techniques, qui sont tout de même assez longues et fastidieuses.

À quelle époque a-t-on commencé à faire figurer le nom du traducteur au générique?

Je m'avance peut-être un peu, mais il me semble que, dans la traduction littéraire, le principe consistant à faire apparaître le nom du traducteur sur une œuvre est très ancien, même s'il est respecté de façon extrêmement variable. Dans le cinéma et l'audiovisuel, il est beaucoup plus récent, non seulement parce que le cinéma est un art jeune, mais aussi parce que la pratique en la matière a toujours été très aléatoire et connaît encore aujourd'hui des hauts et des bas. À toutes les époques, on voit des adaptations non signées, quelles qu'en soient les raisons. II est difficile, aujourd'hui, de consulter des copies doublées et sous-titrées avant la Seconde Guerre mondiale, mais il semble qu'elles ne portaient généralement pas la signature de l'adaptateur.

Dans les années 1930, les seuls noms indiqués dans les versions doublées sont ceux du studio de doublage et des principaux comédiens. Dans les films américains doublés d'après la guerre, on trouve la mention "Dialoguiste " ou "Dialogues de " au générique, à côté du nom du directeur artistique qui a dirigé les comédiens. Pendant une période assez longue, il était d'usage de refaire intégralement les génériques des films doublés pour les franciser. Le nom de l'adaptateur apparaissait donc, non pas en catimini, mais comme une partie intégrante du générique de début ou de fin.

S'agissant du nom de l'adaptateur en sous-titrage, il n'apparaît guère avant les années 1950, et plutôt vers la fin de celles-ci. II est possible qu'il y ait eu des cas avant la guerre, mais je n'en 
ai pas connaissance, sauf cas exceptionnels comme celui de Colette. Techniquement, pourtant, il est très simple de "signer " un sous-titrage ; cela ne nécessite pas de recréer un générique. Les noms de l'adaptateur et du prestataire qui a réalisé techniquement le soustitrage apparaissent sous forme de sous-titres. On a aujourd'hui adopté la convention de faire apparaître cette signature à la toute fin du film, dans les dernières secondes du générique, juste avant le copyright et l'année de production. Depuis une vingtaine d'années, les génériques de fin sont devenus très longs, de sorte que le nom de l'adaptateur s'affiche quand la plupart des spectateurs ont quitté la salle.

Dans les sous-titrages des années 1950, on voit un peu de tout. Le nom de l'adaptateur apparaît tantôt à la fin, tantôt au début du film, pendant le générique, souvent après le titre traduit. II se superpose alors au nom d'autres personnes ayant travaillé sur le film original, le chef opérateur ou le producteur, juste avant le début de l'intrigue proprement dite et des premiers dialogues sous-titrés. Cette pratique était assez courante à la fin des années 1950 et au début des années 1960 .

Ces aléas font qu'il est très difficile d'identifier les traducteurs de versions sous-titrées et doublées. II faudrait explorer les archives de la Sacem, sachant que tous les doublages et soustitrages n'ont sans doute pas été déclarés auprès de cette société d'auteurs. En somme, il reste énormément de choses à rechercher, tant dans ce domaine que du point de vue des choix opérés par les distributeurs de doubler et/ou sous-titrer certains films.

Votre travail est centré sur l'exploitation des films en France, mais il reste encore beaucoup à faire à propos des autres pays. En échangeant avec des traducteurs ou des chercheurs étrangers, on se rend compte qu'il est difficile d'établir ne serait-ce qu'un glossaire commun à plusieurs langues pour la traduction/adaptation audiovisuelle.

Avec les autres pays, il y a en effet des points communs et des différences. Si l'on considère le doublage, les méthodes ne sont pas nécessairement les mêmes dans les autres grands pays de doublage européens que sont l'Allemagne, l'Italie et l'Espagne. L'ironie, c'est d'ailleurs que l'Allemagne, qui est à l'origine de la bande rythmo, ne l'utilise pas. Le procédé Blum a très vite été abandonné outre-Rhin au profit d'une forme de "doublage à l'image ". En Italie et en Espagne, c'est la même chose.

Du point de vue de la terminologie, il y a parfois des incompréhensions ou des inexactitudes. L'appellation " bande rythmo " n'est compréhensible qu'en France. Même dans les autres pays francophones - notamment au Québec -, cette méthode n'est pas utilisée. Dans la définition précise de ce qu'on entend par " doublage ", le critère du respect du synchronisme labial est beaucoup moins important en Italie ou en Espagne, où l'on s'attache davantage à d'autres aspects, tels que la gestuelle des personnages. En sous-titrage, les normes appliquées sont elles aussi variables, par exemple du point de vue de la lisibilité acceptable ou de la durée des sous-titres. 
Une fois que chacun aura mieux défini la terminologie dans sa propre aire linguistique, il sera peut-être possible d'établir des glossaires multilingues ou de se mettre d'accord sur une terminologie multilingue plus cohérente. Ce n'est toutefois pas une certitude.

En raison de ces pratiques différentes, il serait utile d'effectuer dans chaque pays des recherches équivalentes à ce que j'ai entrepris. Les choses évoluent, la jeune génération de chercheurs en traduction audiovisuelle tend à s'intéresser davantage à l'image et au son, sans se focaliser exclusivement sur le texte. II y a aussi un apport des études cinématographiques, de l'histoire du cinéma. Tout cela, pris en compte de façon cohérente, devrait donner des résultats très intéressants à l'avenir. 